\title{
EXPANSIONS OF QUADRATIC FORMS
}

\author{
RUFUS OLDENBURGER
}

1. The problem. A quadratic form $Q$ with coefficients in a field $K$, whose characteristic is different from 2 , is usually given as a linear combination

$$
\sum_{i=1}^{n} a_{i j} x_{i} x_{j}
$$

of products $\left\{x_{i} x_{j}\right\}$, where $\left(a_{i j}\right)$ is symmetric. The sum (1) is one of the type

$$
\sum_{i=1}^{\tau} L_{i} M_{i}
$$

where the $L$ 's and $M$ 's are linear forms. In general the decomposition (1) is not the most economical way of writing $Q$ as a sum of the type (2) in the sense that $\tau$ is a minimum for $Q$. In treating algebras associated with quadratic forms $\mathrm{E}$. Witt ${ }^{1}$ showed that the form $Q$ is equivalent under a nonsingular linear transformation to a decomposition

$$
\sum_{i=1}^{\sigma} y_{i} z_{i}+\sum_{i=1}^{r-2 \sigma} \nu_{i} u_{i}^{2}
$$

where the last sum is a nonzero form, and $r$ is the rank of $Q$. In the present paper we shall show that the minimum $\tau$ for $Q$ is $r-\sigma$. Thus this minimum $\tau$ is determined by the rank $r$ and the "characteristic" $\sigma$ of $Q$. This characteristic ${ }^{2}$ is the maximum number $\sigma$ of linearly independent linear forms $L_{1}, \cdots, L_{\sigma}$ such that the rank of $Q+\lambda_{1} L_{1}^{2}+\cdots+\lambda_{\sigma} L_{\sigma}^{2}$ is the same as the rank of $Q$ for all values of the $\lambda$ 's. The form $Q$ has characteristic $\sigma$ if and only if $Q$ has the canonical splitting $G+H$, where $G$ has characteristic $\sigma$ and rank $2 \sigma$, while $H$ has characteristic 0 and rank $r-2 \sigma$. The form $G$ has a decomposition (2) with $\tau=\sigma$. The decomposition (3) is one such that the first sum is a form $G$ of the type described and the other a form $H$. Thus it will be proved

Presented to the Society, April 18, 1942; received by the editors April 29, 1942.

1 E. Witt, Theorie der Quadratischen Formen in beliebigen Körpern, J. Reine Angew. Math. vol. 176 (1937) p. 35.

2 Rufus Oldenburger, The index of a quadratic form for an arbitrary field, Bull. Amer. Math. Soc. abstract 48-5-162. 
that the decomposition (3) corresponding to a canonical splitting $G+H$ of $Q$ is one with a minimum number of terms.

Like the rank of $Q$ the characteristic of $Q$ has the property that this characteristic changes at most by 1 under addition of a term $\lambda L^{2}$, $L$ linear, to $Q$. We shall prove here that actually the minimum $\tau$, the characteristic $\sigma$ defined above, and the index ${ }^{3}$ (if $K$ is real) possess this property of changing at most by 1 under additions of the type $L M$ to $Q$, where $L$ and $M$ are arbitrary linear forms.

We recall that the rank $r$ of $Q$ is the minimum $\tau$ for which $Q$ can be written as a sum (2), where for each $i$ the forms $L_{i}$ and $M_{i}$ are linearly dependent. Thus both the rank alone, and the rank and characteristic of $Q$, yield minimum properties of expansions of $Q$ invariant under nonsingular linear transformations on the variables in $Q$.

It will be understood throughout the present paper that the coefficients are in a field $K$ of the type specified above. The field $K$ is otherwise unrestricted, except where $K$ is taken to be the real or complex fields.

2. Solution of the minimum problem. The following lemma needs no proof.

LEMMA 1. The characteristic of a quadratic form $Q$ is invariant under nonsingular linear transformations on $Q$.

The lemma to follow was proved elsewhere. ${ }^{4}$

LEMMA 2. The characteristic of a quadratic form $Q$ changes at most by 1 under addition to $Q$ of a term $\lambda L^{2}, L$ linear and $\lambda$ in the given field.

LEMMA 3. The characteristic of a quadratic form $Q$ is at least as great as the characteristic of each form $Q^{*}$ obtained from $Q$ by imposing homogeneous linear relations on the variables in $Q$.

We write $Q$ as in (1), and suppose that $Q^{*}$ is obtained from $Q$ by equating $x_{1}, \cdots, x_{e-1}$ to zero for some $e$. The matrix $A=\left(a_{i j}\right)$ of $Q$ can be written as

$$
\left\|\begin{array}{ll}
* & * \\
* & B
\end{array}\right\|,
$$

where $B$ is the matrix $\left(a_{i j}\right)[i, j=e, \cdots, n]$ of $Q^{*}$, and the asterisks in $A$ indicate minors of $A$. We let $\alpha$ designate the characteristic of

${ }^{3}$ The index of a real quadratic form $Q$ is the number $h$ of + signs in a canonical form $x_{1}^{2}+\cdots+x_{h}^{2}-x_{h+1}^{2}-\cdots-x_{r}^{2}$ to which $Q$ is equivalent.

${ }^{4}$ See the above reference to a paper by $\mathrm{R}$. Oldenburger. 
the form $Q^{*}$. Since $Q^{*}$ has a canonical splitting, as described in $\S 1$, the form $Q^{*}$ is equivalent under a nonsingular transformation to the form

$$
\sum_{i=1}^{\alpha} u_{i} v_{i}+F,
$$

where $F$ is a form of characteristic 0 whose variables are linearly independent of the $u$ 's and $v$ 's. With the form (4) we may associate the symmetric matrix

$$
\left\|\begin{array}{ll}
0 & 0 \\
0 & C
\end{array}\right\|
$$

of order $n-e+1$, where $C$ is a nonsingular minor of the type

$$
\left\|\begin{array}{lll}
0 & 0 & I \\
0 & D & 0 \\
I & 0 & 0
\end{array}\right\|,
$$

the minor $I$ being an identity matrix of order $\alpha$ [arising from the summation in (4) ]. It follows from elementary matrix considerations that there is a nonsingular matrix $N$ such that

$$
N^{\prime} A N=\left\|\begin{array}{ll}
E & 0 \\
0 & 0
\end{array}\right\|,
$$

where $E$ is a nonsingular minor with the shape

$$
E=\left\|\begin{array}{llll}
* & * & * & I \\
* & * & * & 0 \\
* & * & D & 0 \\
I & 0 & 0 & 0
\end{array}\right\|,
$$

and $N^{\prime}$ designates the transpose of $N$. We let $T=T\left(y_{1}, \cdots, y_{n}\right)$ be a quadratic form in $y_{1}, \cdots, y_{n}$ associated in the usual manner with $N^{\prime} A N$. The rank of $T+\lambda_{1} y_{1}^{2}+\cdots+\lambda_{\alpha} y_{\alpha}^{2}$ is the same as the rank of $T$ for all values of the $\lambda$ 's. Since $T$ is equivalent to $Q$ the characteristic of $Q$ is at least $\alpha$.

Suppose now that we impose homogeneous linear relations $Z_{1}=0, \cdots, Z_{s}=0$ on the variables $x_{1}, \cdots, x_{n}$ in $Q$. It is no restriction to take these $Z$ 's to be linearly independent forms. We may therefore use these $Z$ 's and enough of the $x$ 's to obtain a set of linearly independent forms, which we may employ as the $n$ variables in terms of which $Q$ is expressed. By Lemma 1 this change of vari- 
ables leaves the characteristic invariant. Thus the problem which arises when the $Z$ 's are set equal to zero reverts to the above case where $x_{1}=\cdots=x_{e-1}=0$.

THEOREM 1. The minimum $\tau$ for which a quadratic form $Q$ with rank $r$ and characteristic $\sigma$ has the expansion (2), where the L's and M's are linear forms, is $r-\sigma$.

We suppose that $Q$ is written as (2), where $\tau$ is a minimum. If for some $i$ and element $k_{i}$ we have $M_{i} \equiv k_{i} L_{i}$, we write $k_{i} L_{i}^{2}$ in place of $L_{i} M_{i}$ ( $i$ not summed). Thus we can split the sum (2) into $R+S$, where

$$
R=\sum_{i=1}^{s} L_{i} M_{i}, \quad S=\sum_{i=1}^{t} \nu_{i} N_{i}^{2},
$$

$L_{i}$ being linearly independent of $M_{i}$ for each $i$, and the $N$ 's being linear forms. The $L$ 's form a set of linearly independent linear forms, since otherwise we can write $R$ as a sum of products of linear forms with less terms. As in $\S 1$ we write a canonical splitting of $Q$ as $G+H$. Since $Q$ has rank $r$, we may take $Q$ to be a form in $r$ independent variables. Since the rank of $S$ is $t$, we have $t \geqq r-2 s$. If $s<\sigma$, we have $s+t>r-\sigma$, whence the decomposition corresponding to the canonical splitting $G+H$ has less terms than (2). Thus $s \geqq \sigma$, and we can write $s=\sigma+\rho$ for a $\rho \geqq 0$.

We relabel the subscripts on the $L$ 's, $M$ 's, and $N$ 's if necessary so that the forms in the set $\Sigma$, where

$$
\Sigma=\left(L_{1}, \cdots, L_{\sigma+\rho}, M_{1}, \cdots, M_{\zeta}, N_{1}, \cdots, N_{\xi}\right),
$$

yield a minimal basis for the $L$ 's, $M$ 's and $N$ 's. Here $\xi \geqq r-\sigma-\rho-\zeta$. If (2) is a more economical decomposition than that which arises from $G+H$, we have $t \leqq r-\rho-2 \sigma-1$. Now $t \geqq r-\sigma-\rho-\zeta$. These inequalities yield $\zeta \geqq \sigma+1$. We suppose that $\zeta$ satisfies this inequality. We let $Q^{\prime}$ designate the form

$$
Q-\sum_{i=\xi+1}^{t} \nu_{i} N_{i}^{2}
$$

Since by Lemma 2 the characteristic changes at most by 1 under each subtraction with $\nu_{i} N_{i}^{2}$ ( $i$ not summed), the index $\alpha$ of $Q^{\prime}$ is such that

$$
\alpha \leqq \sigma+t-\xi .
$$

Eliminating $t$ with the aid of an inequality relation above, we have $\alpha \leqq \zeta-1$. 
We take the linear forms in the set $\Sigma$ to be the variables in terms of which the form $Q^{\prime}$ above is expressed. Setting $L_{\zeta+1}, \cdots, L_{\sigma+\rho}=0$, we obtain from $Q^{\prime}$ a form $Q^{\prime \prime}$ with index $\zeta$. By Lemma 3, we have $\alpha \geqq \zeta$, giving us a contradiction. It follows that $\tau=r-\sigma$.

For the complex field the characteristic $\sigma$ of $Q$ is $[r / 2]$, whereas for the real field $\sigma$ is the minimum of the indices of $Q$ and $-Q$. These results yield Corollary 1 .

CoRollary 1. For the complex field the minimum number $\tau$ of Theorem 1 is $r-[r / 2]$. For the real field $\tau$ is the maximum of the indices of $Q$ and $-Q$.

Witt proved ${ }^{5}$ that a form is a zero form if and only if the characteristic $\sigma$ of this form is greater than 0 .

COROLlary 2. The form $Q$ of Theorem 1 is a zero form if and only if $\tau \neq r$.

By Theorem 1 the sum (2), where $\tau$ is a minimum, is a sum with the $R$ and $S$ of (5) satisfying $R=G, S=H$, the sum $G+H$ being a canonical splitting of $Q$.

Although addition of a term $L M, L$ and $M$ linear, may change the rank $r$ of $Q$ by 2, this is not true of the index $\sigma$ and $\tau=r-\sigma$ as we shall now prove.

THEOREM 2. Under addition of a term $L M, L$ and $M$ linear, to a quadratic form $Q$, the characteristic $\sigma$, and the minimum number $\tau$ for decompositions of type (2), change at most by 1 .

We write $Q$ as a sum (2) where $\tau$ takes on the minimum value $r-\sigma$, the rank of $Q$ being $r$. We let $\tau^{\prime}, r^{\prime}, \sigma^{\prime}$ designate the analogues for $Q^{\prime}=Q+L M$ of $\tau, r, \sigma$ for $Q$. Since

$$
Q^{\prime}=\sum_{i=1}^{r} L_{i} M_{i}+L M,
$$

we clearly have $\tau^{\prime} \leqq \tau+1$. Thus $\tau$ changes at most by 1 under the addition of $L M$ to $Q$.

We suppose that $L$ and $M$ are linearly independent of each other and of the variables in $Q$ so that $r^{\prime}=r+2$. We write $Q$ as $R+S$, where $R$ and $S$ are given by (5) with $s=\sigma, t=r-\sigma$, whence

$$
Q \equiv \sum_{i=1}^{\sigma} L_{i} M_{i}+\sum_{i=1}^{r-\sigma} \nu_{i} N_{i}^{2}
$$

\footnotetext{
- See the above reference to a paper by E. Witt
} 
The form $R^{\prime}$, where $R^{\prime}=R+L M$, has index $\sigma+1$ and rank $2(\sigma+1)$, from which it follows that $Q^{\prime}$ has the canonical splitting $R^{\prime}+S^{\prime}$ with $S^{\prime} \equiv S$. Thus $Q^{\prime}$ has index $\sigma+1$.

If $L$ and $M$ are taken linearly dependent, or one or both of the forms $L, M$ are restricted to be linear forms in the variables of $Q$, by Lemma 3 we obtain from the form $Q^{\prime}$ of the preceding paragraph a form $Q^{*}$ whose characteristic does not exceed $\sigma+1$. Thus in any case $\sigma^{\prime} \leqq \sigma+1$, whence also $\sigma \leqq \sigma^{\prime}+1$.

If the rank of $Q+L M$ is less than the rank of $Q$, Theorem 2 implies that the characteristic of $Q+L M$ does not exceed that of $Q$, whereas if the addition of $L M$ to $Q$ decreases the rank of $Q$ by 2 , this addition also decreases the characteristic of $Q$.

We have the following analogue of Theorem 2.

THEOREM 3. Under addition of a term $L M, L$ and $M$ linear, to a real quadratic form $Q$, the index of $Q$ changes at most by 1 .

We write $Q$ as the sum $P+N$ of a positive definite form $N$ and a negative definite form $N$, the rank of $Q$ being the sum of the ranks of $P$ and $N$. We suppose that $Q$ is written in any way as a sum $P^{\prime}+N^{\prime}$, where $P^{\prime}$ and $N^{\prime}$ are positive definite and negative definite forms respectively. The index $\alpha$ of $Q$ is the rank of $P$. We let $\beta$ designate the rank of $P^{\prime}$, whence

$$
P^{\prime}=\sum_{i=1}^{\beta} p_{i} P_{i}^{2}
$$

for linear forms $P_{1}, \cdots, P_{\beta}$. We suppose that $\alpha>\beta$. Setting $P_{1}=\ldots=P_{\beta}=0$, we have $P \not \equiv 0$, while $P+N$ is negative definite, a contradiction. Thus $\alpha \leqq \beta$.

The form $L M$ can be written as the difference $R^{2}-S^{2}$, where $R$ and $S$ are linear forms, or one of the terms $R, S$ is zero. The form $Q+L M$ is a sum of the positive definite form $P+R^{2}$, and negative definite part $N-S^{2}$. Since the rank of $P+R^{2}$ differs at most by 1 from the rank of $P$, the index of $Q+L M$ does not exceed $\alpha+1$. It follows that the indices of $Q$ and $Q+L M$ differ at most by 1 .

Illinois Institute of TechNology 